\title{
Elimination and Reduction of Harmful Substances in Cigarette Smoke, Using Nanomaterials: Study Protocol for a Systematic Review
}

Mohammad Hossein Chalak ( $\sim$ mhchalak1994@gmail.com )

Iran University of Medical Sciences https://orcid.org/0000-0002-1894-0805

Soqrat Omari Shekaftik

Iran University of Medical Sciences: Tehran University of Medical Sciences

Hossein Jafari

Zahedan University of Medical Sciences

Hamid Reza Ghaffari

Hormozgan University of Medical Sciences

\section{Sakineh Dadipoor}

Hormozgan University of Medical Sciences

\section{Tsvetan Ivanov Popov}

Missouri S\&T: Missouri University of Science and Technology

\section{Protocol}

Keywords: Nanomaterials (NMs), Intervention, Cigarette Smoke, Harmful Substances, Systematic Review

Posted Date: December 20th, 2021

DOI: https://doi.org/10.21203/rs.3.rs-1173417/v1

License: (1) This work is licensed under a Creative Commons Attribution 4.0 International License.

Read Full License 


\section{Abstract \\ Background}

Cigarette smoke is an aerosol containing more than thousands of chemical compounds, several of which are carcinogens and toxic. Many efforts have been made to use nanomaterials (NMs) to remove and filter toxic substances in cigarette smoke. Due to the characteristics of MLs and their selectivity to different compounds, NMs have a high potential for practical application in reducing harmful compounds in cigarette smoke. The objective of this systematic review is to collect, combine, and analyze the basic intervention research on NMs for removal and reduction of harmful substances in cigarette smoke. This systematic review will determine the most important factors and expected characterizations for the development or commercialization of a new filters. Finally, we will review the challenges of using NMsbased interventions.

\section{Methods}

This protocol is based on the Preferred Reporting Items for Systematic Reviews and Meta-Analyses (PRISMA)-P protocol and Cochrane methodology. The inclusion/exclusion criteria are determined based on PICOTS (Population, Intervention, Comparator, Outcome, Time, Study Design) Framework. The Comprehensive literature searches will be conducted in international databases such as PubMed, Scopus, Science Direct and 3Iranian database, including SID, Iranmedex and Magiran. The quality of the papers and the risk of bias will be assessed using GRADE (Grading of Recommendations, Assessment, Development, and Evaluation) methodology. The results will be presented in the text as well as in a table and figure based on the SWiM guidelines (Synthesis Without Meta-analysis). the Preferred Reporting Items for Systematic Reviews and Meta-Analyses (PRISMA) checklist and guidelines will be tracked to certify a robustness and reproducibility of the process.

\section{Discussion}

A comprehensive analysis of the studies conducted in this field can identify research needs, examine the current and expected situation, provide the necessary parameters for research and development of new filters and ultimately determine the effective priorities for further effective interventions.

\section{Background}

According to $\mathrm{WHO}$, the tobacco epidemic is one of the greatest threat to the public health in the world, causing more than 8 million deaths each year and a global cost of $\$ 1.4$ trillion

$[1,2]$. Statistics show that more than 7 million people died of direct use and about 1.2 million died of indirect use (environmental tobacco smoke) [2,3] Cigarette smoke has cause many chronic diseases and 
cancers [3]. Many efforts have been made to reduce the epidemics caused by smoking, but no significant success has been achieved so far. Therefore, in order to reduce the impacts of these epidemics, it is very necessary to reduce or eliminate harmful compounds in cigarette smoke [4].

Cigarette smoke is an aerosol including more than thousands of chemical compounds including nicotine, tar, particulate matters (PM), heavy metals (arsenic, chromium, cadmium, lead, etc.), polycyclic aromatic hydrocarbons, as well as several carcinogens and other toxic elements. they exist in the form of particulate matters and vapor. Inhaling this toxic aerosol of cigarette is a significant part for development of many diseases such as cancer, asthma and hypertension [4-6]. Furthermore, nicotine is one of the main components of cigarette smoke, in addition to being highly addictive, it has also dangerous side effects. An common cigarette includes about $2 \mathrm{mg}$ absorbed nicotine, which is too much for an unrestricted smoker [7]. therefore, it is necessary to diminish its concentration in cigarette smoke to be safe for use. Provide a new approach to reduce harms caused by cigarette smoke can be an important health issue and international scientific consideration [8]. Nano-materials due to special features and selective sensitivity to different combinations has potential applications for use in reducing the harmful substances in tobacco smoke [9]. In this regard, some nanostructured adsorbents have been considered to remove toxic chemicals/compounds caused by cigarette smoke [6]. As an extraordinary class of materials, nanomaterials includes a wide range of samples with at least one dimension in the range of 1 to 100 nanometers. through the logical design of this material, an extremely high surface area can be created. Nanomaterials can be produced with outstanding magnetic, electrical, optical, mechanical and catalytic properties that are significantly different from their main counterparts. The physical and chemical properties of nanomaterials can be arbitrarily adjusted by precisely modifying and controling thesize, shape, synthesis conditions and performance [10].Recently, nanotechnology tools have been widely used tofilter and remove of contaminants from air, soil, solid waste and wastewater. It is known that nanoparticles between 1 and $100 \mathrm{~nm}$ can be used in several filtration and decontamination processes that allow nanotubes, nano-sheets and nano-layers to be used as filtration materials [11]. Several studies have been performed on the use of nanomaterials to remove and filter toxic substances from cigarette smoke. For this, different types of nanomaterials with specific physical and chemical properties have been used. The nanomaterials have been synthesized and evaluated using different methods. For example, carbon nanomaterials have been used to remove toxic substances in cigarette smoke in various ways in experimental and theoretical studies. Each material has different removal efficiency for toxic compounds, and the removal of toxic compounds is also different (Figure 1). In several studies investigating the use of nanomaterials to remove toxins from cigarette smoke, several factors have been studied that affect the removal of toxic substances from cigarette smoke and the safety of new nano-based filters. In these studies, nanomaterials are added to cigarette filters in various forms (powders, suspensions, etc.). In addition, various methods and materials have been used to add substances that can react with components of cigarette smoke to create toxic compounds that pose new risks to smokers $[8,9,12-14]$. These nanomaterials may also affect the environment when disposing of cigarette filters. Figure 2 shows the conceptual framework of the factors that influence the design of the filter. In literature different interventions performed using nanomaterials and each intervention has shown 
different results and efficiency according to the type of nanomaterial, special properties of nanomaterials, and different methods of synthesis. It is essential to conduct a comprehensive study of interventions using nanomaterials to remove toxins from cigarette smoke. to the best of our knowledge, this study will be the first study to review these interventions.

We hope to identify the need to develop new filters or conduct further research or commercialize new filters based on previous research. We will also summarize the results of these 2 years of literature and determine the most important factors and expected characteristics required for the development of a new filter or its commercialization. Finally, the challenges of using these nano-based filters for cigarette smoke restoration will be reviewed.

\section{Objective and questions of this Review}

The objective of this systematic review study is to collect, combine, and analyze the researches on nanomaterials that has been used for removal and reduction of harmful substances in cigarette smoke. After running in the systematic review, we hope to answer the following questions:

1. is it possible to commercialize a new safe filter based on past studies?

2. What types of nanomaterials have been studied during the development of new filters?

3. could the nanomaterials used increase the efficiency of cigarette filters?

4. Are further studies needed to make the filter safer and more cost effective?

5. Where are we in developing an efficient, safe and cost-effective filter?

6. What are the important factors which should be considered when developing a new filter?

\section{Methods/design}

the methods are being developed according to the Preferred Reporting Items for Systematic Reviews and Meta-Analyses (PRISMA)-P protocol and Cochrane methodology. The inclusion/exclusion criteria are listed below base on PICOTS (Population, Intervention, Comparator, Outcome, Time, Study Design) Framework [15].

\subsection{Inclusion Criteria}

\subsubsection{Population}

This systematic review will include all types of filters traditionally developed using additives (nanomaterials) and filters newly designed with Nano-based materials to remove toxic substances from cigarette smoke.

\subsubsection{Intervention}

Any kind of traditional filters or conventional commercialization that has used any species of nanomaterials to remove any toxic substance. We will also consider any new type of filter designed 
based on any type of nanomaterial. Studies that have used any new and cost-effective or traditional and costly methods for the synthesis of nanomaterials to remove toxic substances from cigarette smoke.

\subsubsection{Comparator}

The results related to the removal of toxic substances from cigarette smoke by nanomaterials and the characteristics of the designed filters will be compared with the existing conventional and commercialized filters such as activated carbon and zeolite.

\subsubsection{Outcomes}

Primary Outcomes:

1. The names of the nanomaterials used

2. Characteristics (Length, diameter, pore size and pore volume, surface area, physical characteristics) of the nanomaterial used (For experimental studies )

3. Characteristics (absorption energy, diameter, Geometric strength Stability) (for theoretical studies)

4. The type of theory used such as adsorption energy and Bader charge transfer (for theoretical studies)

5. Removal efficiency of toxic substances using new nanomaterials-based fillers (For experimental studies )

6. Methods used for synthesis of Nanomaterial

7. Smoker machine type and compliance with standard (For experimental studies)

8. The physical or chemical process of adsorption or both (For experimental studies)

9. Performing tests to check the correct synthesis of nanomaterials (For experimental studies)

Secondary Outcomes:

1. The cost-effectiveness of filters usage for commercialization

2. The potential hazards of the new filter designed for the smoker

3. Molecular structure and shape of nanomaterials

4. The filter pressure drops (For experimental studies)

5. Molecule-nanotube interaction and cigarette smoke

Only the primary outcomes will be considered for selection of studies and we will consider secondary outcome if reported.

\subsubsection{Adverseevents}

Any facts of harmful effects of the intervention, including the following: 
1. Reports of toxicity or adverse effect to the smokers as a new threat through using additive (NMs) to cigarette filter

2. Reports of toxicity or adverse effect to the smokers as a new threat through using binding material for putting NMS to cigarette filter

3. Reports of environmental impacts as a new threat through disposing nanomaterial-based cigarette filter

\subsubsection{Time}

Since the 1950s, researchers have proposed many methods to reduce the harmful impacts of toxic compounds in cigarette smoke [8]. The first attempts to remove toxic substances using nanomaterials were made early two decades ago $[13,17]$. Therefore, we will include original research (experimental and theoretical studies) published in English and Persian from the last 15 years to consider a comprehensive range of studies.

\subsubsection{Study Design}

We will include only interventional study that developed or design a new filter by additive nanomaterial. In this review, we will focus on the advancement in Nano material-based intervention and removal of cigarette smoke harmful agent by employing these Nano materials, considering both theoretical and experimental studies for the purpose of cigarette smoke remediation.

\section{Exclusion Criteria}

All type of material which does not comply with inclusion criteria will be carefully reviewed and omitted. We also will remove the literature from other languages than English and Persian. We will exclude studies that their full doesn't exist. We only included studies that used nanomaterials to remove cigarette smoke aerosols and duplicates of studies articles will have removed by title.

\section{Review Team Members}

Two investigators (MHC and SD) will guidance this systematic review and supervise all aspects of the study including protocol formation, data extraction and analysis and interpretation, manuscript writhing, preparation and submission. An occupational and safety expert with knowledge and experience in systematic reviews (SO) will extend the search strategy. he will also assist in reviewing protocols, results and manuscripts. Tow expert in environmental health engineering ( $\mathrm{r} \mathrm{Gh}$ and $\mathrm{Dr} \mathrm{Po}$ ) familiar with review studies will assist in monitoring and reviewing results and manuscripts. Two people (MHC and $\mathrm{HJ}$ ) will be in charge of searching and extracting the results and the results will be confirmed and finalized by 3 observers ( $\mathrm{Dr}$ GH, Dr SD and Dr Po). Also, the combination of the extracted results will be finalized under the supervision of 3 people ( $\mathrm{Dr} \mathrm{D}, \mathrm{Dr} \mathrm{GH}$, Dr Po).

\section{Data Sources and Search Strategy}


The complete search strategy is given in the attached file. The systematic review will be performing systematically with items suggested for systematic review studies and meta-analysis (9). We will be carrying out a comprehensive literature searches on PubMed, Scopus, Science Direct and manually search the 3 Iranian databases including, SID, Iranmedex and Magiran. All published and unpublished studies and conferences will be searched, but we will focus more on peer-reviewed studies. After identifying the main articles, we will use the "Related articles" and "citations" options in Google Scholar and ResearchGate to ensure the search results. Also, we will check the references of the main articles as another way to ensure the search method. We will use EndNote software (Clarivate Analytics, PA, USA) for reference management. Appendix one describes this search strategy.

\subsection{Study Selection Process}

Title and abstract screening will be accomplished separately by the two analysts (MHC, HJ). After screening the title and abstract, full texts of the remaining articles will be also reviewed by MHC, $\mathrm{HJ}$ individually and the final articles will be selected based on inclusion criteria. Then screened studies will be shared with the supervisors and the final articles will be selected after discussion and consensus. We will report the numbers of articles included and excluded in each stage including, title and abstract screening and full text review, and exclusion in a PRISMA flow diagram (see Figure 1).

\subsection{Dealing with Companion and Duplicate Publications}

We will remove duplicate studies firstly by EndNote software and while reviewing references.

\subsection{Data Extraction}

Two reviewers (M.CH) will independently extracted data of the eligible articles using a form designed by the author (M.CH) and reviewed and approved by three supervisors ( $\mathrm{Dr} \mathrm{D}, \mathrm{Dr} \mathrm{GH}, \mathrm{Dr} \mathrm{Po}$ ). They resolved the disputes by discussion and, if it will necessary, they will receive help of three supervisors ( $\operatorname{Dr} \mathrm{D}, \mathrm{Dr} \mathrm{GH}, \mathrm{Dr}$ Po). A data extraction form which is presented as Supplementary file 2, will be developed and next pilottested on six randomly picked studies. The following data will be obtained for each elected study: title, authors, country, contact information of corresponding author, journal (or source for reports), study type, year of publication, intervention type, NM species, composition of NM (Adsorbent), dosage (mg), target or type of , removal efficiency (\%), absorption capacity, physical and chemical properties of nanomaterials, the type of theories used in theoretical studies, tests performed on nanomaterials, synthesis method and main conclusions with point measures of variance (standard errors, $95 \% \mathrm{Cl}$ and/or $\mathrm{p}$-values ).

\subsection{Dealing with Missing Data}

In case of missing data or inconsistent outcomes over reports of a particular study, we will contact the corresponding author via email to gather extra accurate results or extra information. A maximum of two communication attempts will be performed. If we cannot decide the issues with the data after communicating the authors, we will investigate the possible data and explain the potential impact of the missing data. 


\subsection{Data Synthesis}

We will carry out a systematic descriptive synthesis of the data obtained from all involved studies. The comparisons will focus on (1) categorizing NMs species that have been studied in the development of new filters, (2) the removal efficiency of the developed filters (3) type of Nano materials and synthesize method (4) physical and chemical characteristic of NMs (5) the process of the physical or chemical adsorption of toxic substances. Firstly, immediate outcomes will be examined and later the delayed consequences, if they have been evaluated. we will present results in the text as well as in a table and figure based on the SWIM guidelines (Synthesis Without Meta-analysis) [18]. We will not perform the meta-analyses given the significant heterogeneity of both the interventions and results. We also will track the Preferred Reporting Items for Systematic Reviews and Meta-Analyses (PRISMA) checklist and guidelines to certify a strong and replicable process [19].

\subsection{Quality of evidence and risk of bias assessment}

The quality of the papers and risk of bias will be assessed using GRADE (Grading of Recommendations, Assessment, Development, and Evaluation) methodology [20]. In this method, study design, risk of bias, imprecision, inconsistency, indirectness, and magnitude of effect, are the criteria for quality assessment. based on the final score, the quality of each paper will be categorized as very low ( -14 to -8$)$ or low ( 0 to -7) or moderate (1 to 7 ) or high (8 to 14 ) [21].

In GRADE method risk of bias instrument, mentioned that bias could due to: (1) confounding, (2) selection of participants, (3) Classification of exposures, (4) Departures from intended exposures, (5) Missing data, (6) Measurement of outcomes, and (7) Selection of reported results. Judgment for each item is based on adjectives term("Low," "Moderate," "Serious," or "Critical."). To reach a judgment for each risk of bias, firstly, one or more questions will answer with "Yes," "Probably yes," "Probably no," or "No." Finally, the study-level bias will be assess [22].

\section{Discussion}

A comprehensive analysis of the studies conducted in this field can identify research needs and examine the current and expected situation, and ultimately lead to the determination of effective priorities for more effective interventions.

Evaluation of the removal efficiency of toxic compounds by interventions performed using nanomaterials in filters and identifying the main factors affecting the removal efficiency plays an important role to provide the necessary parameters in the research and development of new filters. Also, in selecting the best nanomaterials for commercial making and using cigarette filters can be very helpful. For example, examining nano-based filters to remove the nicotine and examining the removal efficiency and characteristic of these filters can be helpful in selecting the high-efficiency nicotine removal filters. Also, it can help to develop new filters to significantly reduce nicotine as the main cause of addiction in cigarette 
smoke. It would be a step towards reducing the consequences of this substance in consumers and reducing dependency on this substance.

Identification of different species used to remove toxic compounds, summary, analysis and comprehensive comparison of physical and chemical properties of these species can help researchers to choose the type of nanomaterial in their interventions and fabrication of nanocomposites. Also, reviewing, comparing and summarizing different methods of nanomaterial synthesis in interventional studies can help to choose the type of synthesis method in future studies. Also, researchers can choose the most cost-effective method for synthesis. Therefore, if a high-efficiency filter is developed, its production would be economically viable and it will be possible to commercialize and widely use it in the tobacco industry.

\section{Conclusions}

This systematic review will compile, collects, combines, and analyzes current research on the use of different types of nanomaterials in the removal of toxic substances from cigarette smoke. These results will be useful for researchers and tobacco factories and will help researchers to consider the most important factors when developing conventional filters to achieve the best and most practical results. Thus, we deem that the result of present study will establish a new avenue for the development or Commercialization of an efficient and economical, safe filter for the cigarette.

\section{Declarations}

\section{Authors' contributions}

Mhc leads the conception and design of the systematic review with the intellectual and technical assistance of SO, SD, and HRG. Mhc drafted the protocol manuscript, and all authors (SD, HRG, SO, HJ, and TIP) provided feedback and comment as well contributed to the revisions. All authors review and approve the final manuscript before submission.

\section{Funding}

This project did not take any particular grant from a funding organization.

\section{Availability of data and materials}

Not applicable.

\section{Ethics approval and consent to participate}

Not applicable.

\section{Consent for publication}


Consent gained from all authors for publication.

\section{Competing interests}

The authors declare that they have no competing interests.

\section{References}

1. World Health Organization. WHO report on the global tobacco epidemic, 2021 addressing new and emerging products. World Health Organization. 2021. Available from:

https://www.who.int/publications/i/item/9789240032095.

2. World Health Organization. (2017). WHO report on the global tobacco epidemic, 2017: monitoring tobacco use and prevention policies. World Health Organization. https://apps.who.int/iris/handle/10665/255874.

3. Onor IO, Stirling DL, Williams SR, Bediako D, Borghol A, Harris MB, et al. Clinical effects of cigarette smoking: epidemiologic impact and review of pharmacotherapy options. International journal of environmental research and public health. 2017;14(10):1147.

https://doi.org/10.3390/ijerph14101147.

4. Pandey SK, Vishwakarma PK, Yadav SK, Shukla P, Srivastava A. Multiwalled Carbon Nanotube Filters for Toxin Removal from Cigarette Smoke. ACS Applied Nano Materials. 2019;3(1):760-771. https://pubs.acs.org/doi/10.1021/acsanm.9b02277.

5. Kim SY, Sim S, Choi HG. Active, passive, and electronic cigarette smoking is associated with asthma in adolescents. Scientific reports. 2017;7(1):1-8. https://doi.org/10.1038/s41598-017-17958-y.

6. Yoosefian M, Pakpour A, Zahedi M. Carboxylated single-walled carbon nanotubes as a semiconductor for adsorption of acrylamide in mainstream cigarette smoke. Physica E: Lowdimensional Systems and Nanostructures. 2020;124:114299.

https://doi.org/10.1016/j.physe.2020.114299.

7. Mayer B. How much nicotine kills a human? Tracing back the generally accepted lethal dose to dubious self-experiments in the nineteenth century. Archives of toxicology. 2014;88(1):5-7. https://doi.org/10.1007/s00204-013-1127-0.

8. Cao J, Ma X, Yang A, Xu W. Preparation of cellulose acetate/nano-SiO2 composites and their application in filtration of cigarette smoke. Polymers and Polymer Composites. 2006;14(1):65-71. https://doi.org/10.1177/096739110601400106.

9. Yoosefian M, Pakpour A, Etminan N. Nanofilter platform based on functionalized carbon nanotubes for adsorption and elimination of Acrolein, a toxicant in cigarette smoke. Applied Surface Science. 2018;444:598-603. https://doi.org/10.1016/j.apsusc.2018.03.108.

10. Baig N, Kammakakam I, Falath W. Nanomaterials: a review of synthesis methods, properties, recent progress, and challenges. Materials Advances, 2021;2(6):1821-1871.

https://doi.org/10.1039/D0MA00807A.

Page 10/13 
11. Gulati A, Kakkar R. Graphene-based adsorbents for water remediation by removal of organic pollutants: Theoretical and experimental insights. Chemical Engineering Research and Design. 2020;153:21-36. https://doi.org/10.1016/j.cherd.2019.10.013.

12. Pandey SK, Vishwakarma PK, Yadav SK, Shukla P, Srivastava A. Multiwalled Carbon Nanotube Filters for Toxin Removal from Cigarette Smoke. ACS Applied Nano Materials. 2020;3(1):760-771. https://pubs.acs.org/doi/10.1021/acsanm.9b02277.

13. Chen Z, Zhang L, Tang Y, Jia Z. Adsorption of nicotine and tar from the mainstream smoke of cigarettes by oxidized carbon nanotubes. Applied Surface Science. 2006;252(8):2933-2937. https://doi.org/10.1016/j.apsusc.2005.04.044.

14. Chai Y, Fei Y, Hao J, Wu D, Zhao Q, Wang X, et al. Application of novel graphene nanomaterial to reducing $B[a] p$ and phenol in mainstream cigarette smoke. Acta Tabacaria Sinica. 2016;22(5):19-25. 10.16472/j.chinatobacco.2015.532

15. Amir-Behghadami M, Janati A. Population, Intervention, Comparison, Outcomes and Study (PICOS) design as a framework to formulate eligibility criteria in systematic reviews. Emerg Med J. 2020 Jun;37(6):387. 10.1136/emermed-2020-209567.

16. Hoffmann D, Hoffmann I, El-Bayoumy K. The less harmful cigarette: a controversial issue. A tribute to Ernst L. Wynder. Chemical research in toxicology. 2001;14(7):767-790. https://doi.org/10.1021/tx000260u.

17. Campbell M, McKenzie JE, Sowden A, Katikireddi SV, Brennan SE, Ellis S, et al. Synthesis without meta-analysis (SWiM) in systematic reviews: reporting guideline. bmj. 2020;368 https://doi.org/10.1136/bmj.16890.

18. Moher D, Shamseer L, Clarke M, Ghersi D, Liberati A, Petticrew M, et al. Preferred reporting items for systematic review and meta-analysis protocols (PRISMA-P) 2015 statement. Systematic reviews. 2015;4(1):1-9. https://doi.org/10.1136/bmj.g7647.

19. Guyatt G, Oxman AD, Akl EA, Kunz R, Vist G, Brozek J, et al. GRADE guidelines: 1. IntroductionGRADE evidence profiles and summary of findings tables. Journal of clinical epidemiology. 2011;64(4):383-394. 10.1016/j.jclinepi.2010.04.026.

20. Shekaftik S Omari, Nasirzadeh N. 8-Hydroxy-2'-deoxyguanosine (8-OHdG) as a biomarker of oxidative DNA damage induced by occupational exposure to nanomaterials: a systematic review. Nanotoxicology 2021:1-15. 10.1080/17435390.2021.1936254.

21. Morgan RL, Thayer KA, Santesso N, Holloway AC, Blain R, Eftim SE, et al. A risk of bias instrument for non-randomized studies of exposures: a users' guide to its application in the context of GRADE. Environment international 2019;122:168-184. 10.1016/j.envint.2018.11.004.

\section{Figures}




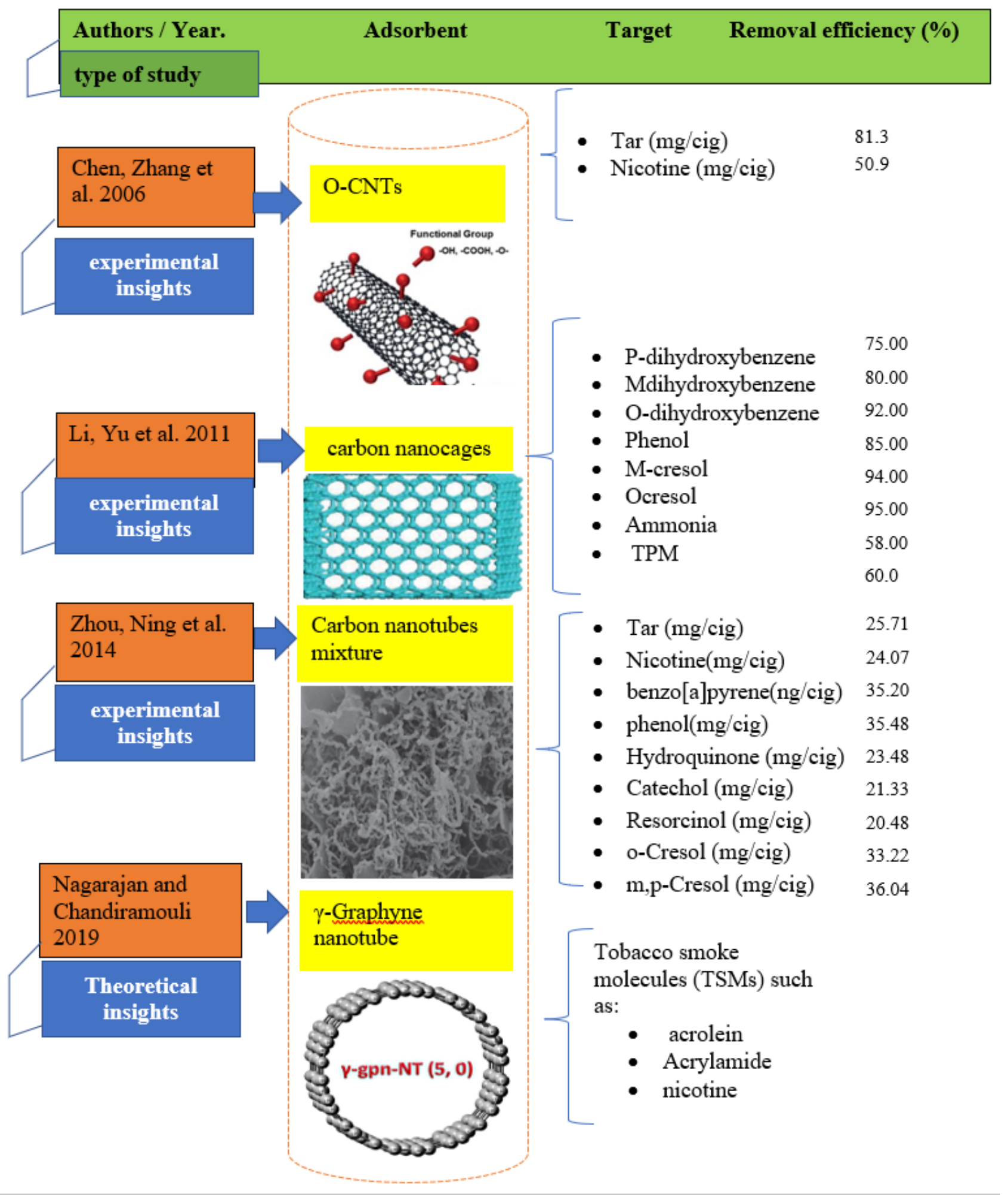

\section{Figure 1}

Use of carbon nanomaterials in various studies 

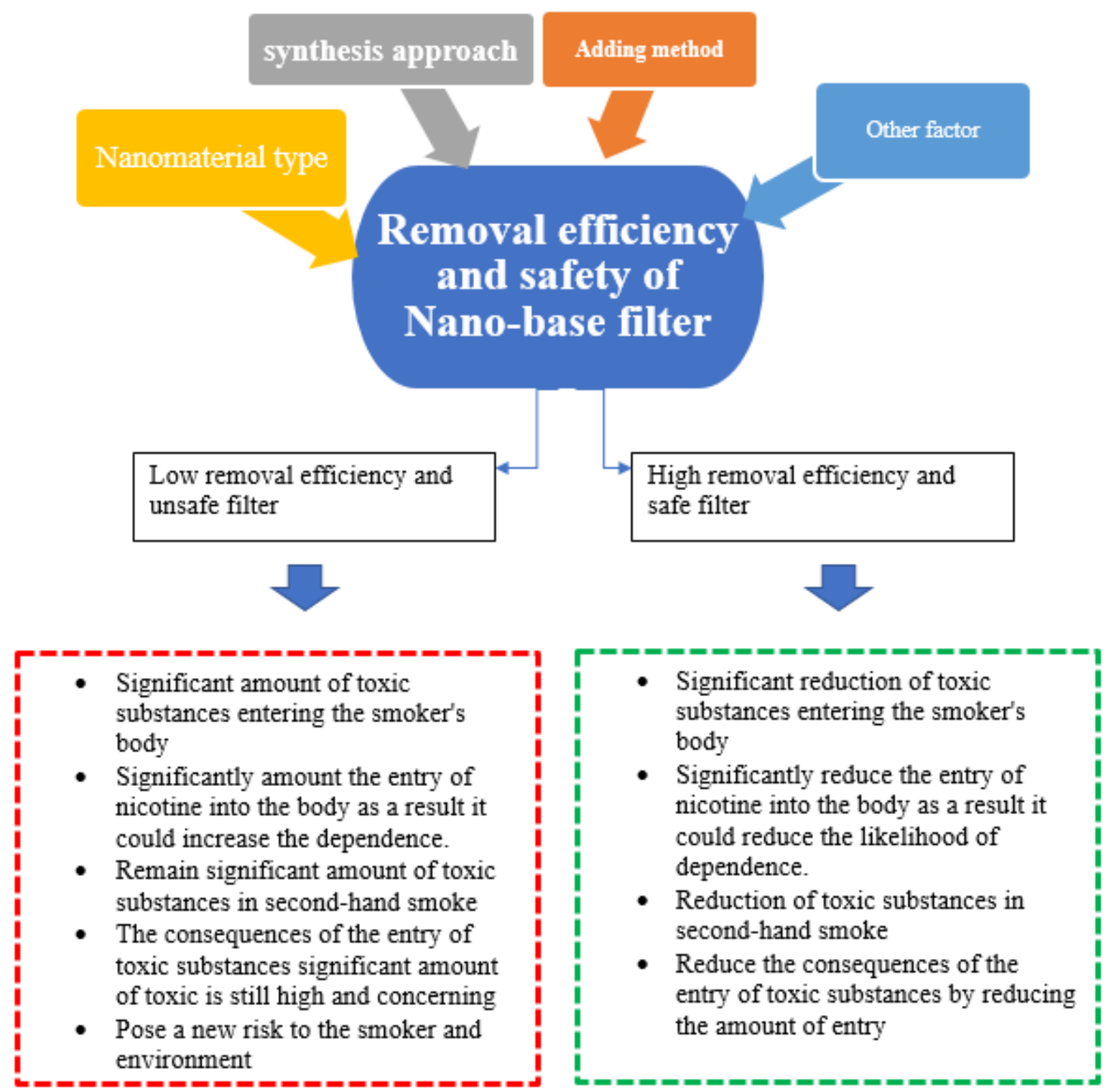

Figure 2

Conceptual framework of the effective factors in efficiency and safety of Nano base-filters in the area of Reduction of harmful substances in cigarette smoke

\section{Supplementary Files}

This is a list of supplementary files associated with this preprint. Click to download.

- Supplementaryfile1.docx

- Supplementaryfile2.docx 